\title{
Non-traumatic Abdominal Surgical Emergencies in Children
}

\author{
Shazim Harunani, Kennedy Imbaya, Tenge Kuremu
}

Moi University, Eldoret

Correspondence to: Dr. Harunani Shazim, PO Box 82647, Mombasa; email: dr s.harunani@hotmail.com

\begin{abstract}
Background: In developing countries, non-traumatic childhood abdominal surgical emergencies (NTCASE) are associated with high morbidity and mortality and include acquired diseases like acute appendicitis, intussusception and congenital abdominal anomalies such as gastroschisis. This study sought to identify the etiologies and outcomes of NTCASE at Moi Teaching and Referral Hospital (MTRH), Kenya. Methods: A prospective descriptive study that involved children below 18 years operated on for non-traumatic abdominal surgical emergencies between January 2015 and January 2016. Relevant data on clinical presentation, duration of onset of symptoms, investigations and intraoperative findings were entered into the data collection sheet. Daily follow-up was done until discharge or in-hospital death, and outcomes recorded. Results: A total of 140 patients were studied. Age of the patients ranged from 1 day to 17 years, with a mean of $3.0(S D \pm 2.9)$ years. Children below 2 years constituted $72.9 \%$ of the study participants. The male to female ratio was 2.1:1. The
\end{abstract}

\section{Introduction}

Non-traumatic childhood abdominal surgical emergencies(NTCASE) are common and theiretiologies many. Some etilogies are acquired gastrointestinal diseases such as acute appendicitis, intussusception and perforation peritonitis, and congenital anomalies such as anorectal malformations and gastroschisis. In young children, aetiologies have been reported to have high mortality in developing countries (1). Among the leading causes of high morbidity and mortality in developing countries are delayed presentation and poverty (2). Electrolyte derangements occur more often in young children and may delay management. Other factors affecting mortality include ASA classification and haemoglobin level. Children constitute more than $40 \%$ of the population in most developing countries and therefore deserve a share of robust surgical care; thus, surgical services should be planned to reduce mortalities associated with these abdominal emergencies (2). Data on NTCASE is scarce at Moi Teaching and Referral most common aetiologies were intussusception (17.9\%) and gastroschisis (17.9\%). The complication rate was $17.9 \%(n=25)$, average length of hospitalization $8.6 \pm 7.6$ days, and in-hospital mortality $20 \%$. Conclusion: The commonest aetiologies of NTCASE in MTRH are intussusception and gastroschisis. Mortality and morbidity rates are high. Factors co-related with mortality in patients with NTCASE are deranged potassium level, ASA classification grade 3 and 4, neonates, normal haemoglobin level, duration of illness presentation $<24$ hours, and development of complications.

Keywords: Non-traumatic childhood abdominal surgical emergencies, Etiologies, Outcomes, Children

Ann Afr Surg. 2020; 17(1): 3-6.

DOI: http://dx.doi.org/10.4314/aas.v17i1.2

Conflicts of Interest: None

Funding: None

(C) 2020 Author. This work is licensed under the Creative Commons Attribution 4.0 International License.

Hospital (MTRH) and in Kenya. This study will act as a backbone of comparing data from MTRH with other institutions. This study aims to elucidate the various etiologies and immediate postsurgical outcomes in children managed for non-traumatic abdominal surgical emergencies at the MTRH and the factors correlated with mortality.

\section{Methodology}

A prospective descriptive study of children aged below 18 years presenting with non-trauma related abdominal emergencies at MTRH (surgical wards and newborn unit) between January 2015 and January 2016.

The sample size was determined using Fisher et al.'s statistical formula (3):

$$
n=\mathrm{Z} 2 \mathrm{pq} / \mathrm{d} 2
$$

where $n=$ desired sample size (when population is greater than 10,000), $Z=$ the standard normal deviate set at 1.96 which corresponds to $95 \%$ confidence level, $\mathrm{p}=$ characteristic of the study population $(10.1 \%$ 
mortality rate from previous data), $\mathrm{q}=1-\mathrm{p}$ (in this case $\mathrm{q}=1.0-0.101$ ), and $\mathrm{d}=$ the degree of accuracy desired, set at $5 \%$ or 0.05 corresponding to the 1.96 . A sample size of 140 was calculated.

Inclusion criteria were children operated on for nontraumatic abdominal surgical emergencies at the MTRH and children with a consent and assent for those above 7 years. Exclusion criteria were patients who had been operated on elsewhere and then referred to MTRH and patients with obvious comorbidities.

The starting point for data collection was in the surgical wards and new born unit after patients were identified from the theatre register. Patients who met the inclusion criteria were consecutively enrolled in the study. Data collection was conducted by the investigators and entailed filling a questionnaire on clinical presentation, duration of onset of symptoms, investigations, supportive management and intraoperative findings as per case notes.

Outcome evaluation parameters included complications, hospital stay and death. Daily follow-up was done until discharge or in-hospital death, either of which was taken as the end point of the study. All filled questionnaires were checked for completeness, and coded accordingly. Data was entered into an Access database and exported to SPSS version 20.0 for analysis. Continuous data were analysed using means and standard deviation (SD). Categorical data were presented as frequency tables and charts. For binary data, Chi-square test and Fisher's exact test were used for analysis. A p value of $<0.05$ was considered significant.

\section{Ethical statement}

This study was approved by IREC, FAN 1277. Approval from the MTRH was also sought and granted.

\section{Results}

Patients' ages ranged from 1 day to 17 years, with a mean of 3.0 and SD of \pm 2.9 years. Neonates comprised $67(65.7 \%)$ out of the 102 children. The male to female ratio was 2.1:1. Table 1 shows the diverse aetiologies identified. The average length of hospitalization and SD was 8.6 \pm 7.6 days, ranging from 1 day to 35 days. Overall in-hospital mortality was 20\% (28 patients) with 24 being neonates (Table 2).

\section{Outcome of NTCASE}

The overall complication rate was $17.9 \%(n=25)$. Neonates constituted 19 of these 25 patients. Table 3 shows the specific complications identified.
Table 1. Etiology of NTCASE

\begin{tabular}{lrc}
\hline Aetiology of NTCASE & $\begin{array}{l}\text { Frequency } \\
(\%)\end{array}$ & $\begin{array}{l}\text { Mean age of } \\
\text { patients }\end{array}$ \\
\hline Gastroschisis & $25(17.9)$ & 1.64 days \\
Intussusception & $25(17.9)$ & 1.57 years \\
Anorectal malformations & $15(10.7)$ & 4 days \\
Small bowel atresia & $15(10.7)$ & 9.8 days \\
Appendicular abscess & $7(5.0)$ & 10.57 years \\
Acute appendicitis & $11(7.9)$ & 9.36 years \\
Inguinal hernias & $9(6.4)$ & 2.16 years \\
Ileal perforations & $5(3.6)$ & 4.68 years \\
Others* & $28(19.9)$ & 3.67 years \\
\hline
\end{tabular}

* umbilical hernia, omphalocele, pyloric stenosis, Hirschsprung's, necrotizing enterocolitis, ascariasis

Table 2. Case-specific mortality

\begin{tabular}{lc}
\hline Etiology of NTCASE & Case fatality frequency $(\%)$ \\
\hline Gastroschisis & $11(44)$ \\
ARM & $5(33.3)$ \\
Small bowel atresia & $5(33.3)$ \\
Ileal perforation & $3(60)$ \\
Colon atresia & $1(100)$ \\
NEC & $1(50)$ \\
Intussusception & $2(8)$ \\
Total & $28(20)$ \\
\hline
\end{tabular}

Table 3. Complications identified

\begin{tabular}{lc}
\hline Complication & Frequency (\%) \\
\hline Septicemia & $19(76)$ \\
Wound dehiscence & $2(8)$ \\
Burst abdomen & $1(4)$ \\
Electrolyte derangement & $1(4)$ \\
Prolapsed stoma & $1(4)$ \\
Enterocutaneous fistula & $1(4)$ \\
\hline Total & $25(100)$ \\
\hline
\end{tabular}

\section{Factors correlated with mortality}

Development of complications, duration of illness (<24 hours), ASA classification (grade 3, 4), deranged potassium levels, normal hemoglobin level $(>12 \mathrm{~g} / \mathrm{dL})$; neonates had significant correlation with mortality (Table 4).

\section{Discussion}

Congenital anomalies were more common; average age of children was low compared with studies from India, Nigeria and Ghana (4-6). The tertiary nature of services offered may explain the difference in etiology and mean age when compared with studies from other developing economic regions. Gastroschisis, a serious congenital 
Table 4. Correlation between various variables and eventual outcome

\begin{tabular}{|c|c|c|c|}
\hline \multirow[b]{2}{*}{ Variable } & \multicolumn{2}{|c|}{ Outcome } & \multirow[b]{2}{*}{$p$ value } \\
\hline & Alive (\%) & Dead (\%) & \\
\hline \multicolumn{4}{|c|}{ Presence of complications } \\
\hline No & $99(86.1)$ & $16(13.9)$ & \\
\hline Yes & $13(52)$ & $12(48)$ & 0.0001 \\
\hline \multicolumn{4}{|l|}{ Sex } \\
\hline Male & $78(82.1)$ & $17(17.9)$ & \\
\hline Female & $34(75.6)$ & $11(24.4)$ & 0.373 \\
\hline \multicolumn{4}{|c|}{ Any antibiotic given } \\
\hline Yes & $98(79.7)$ & $25(20.3)$ & \\
\hline No & $14(82.4)$ & $3(17.6)$ & 0.794 \\
\hline \multicolumn{4}{|l|}{$W B C$} \\
\hline Normal & $59(80.8)$ & 14(19.2) & \\
\hline Abnormal & $49(77.8)$ & $14(22.2)$ & 0.576 \\
\hline \multicolumn{4}{|c|}{ Temperature at admission } \\
\hline Normal & $79(70.5)$ & $23(29.5)$ & \\
\hline High & $33(86.8)$ & $5(13.2)$ & 0.217 \\
\hline \multicolumn{4}{|l|}{ Gut viability } \\
\hline Viable & $102(80.3)$ & $25(19.7)$ & \\
\hline Gangrene & $10(76.9)$ & $3(23.1)$ & 0.771 \\
\hline \multicolumn{4}{|c|}{ ASA classification } \\
\hline 1,2 & $100(85.5 \%)$ & $17(14.5)$ & 0.0003 \\
\hline 3,4 & $12(52.2)$ & $11(47.8)$ & \\
\hline \multicolumn{4}{|c|}{ Duration of illness } \\
\hline$<24$ hours & $28(66.7)$ & 14(33.3) & \\
\hline$>24$ hours & $84(85.7)$ & 14(14.3) & 0.0098 \\
\hline \multicolumn{4}{|c|}{ Referral status } \\
\hline Referrals & $71(77.2)$ & $21(22.8)$ & 0.247 \\
\hline Home & $41(85.4)$ & $7(14.6)$ & \\
\hline \multicolumn{4}{|c|}{ Potassium level } \\
\hline Normal & $86(87.8)$ & 12(12.2) & \\
\hline Abnormal & $26(61.9)$ & 16(38.1) & 0.0005 \\
\hline \multicolumn{4}{|l|}{ Sodium level } \\
\hline Normal & $85(80.2)$ & $21(19.8)$ & \\
\hline Abnormal & $27(79.4)$ & $7(20.6)$ & 0.921 \\
\hline \multicolumn{4}{|c|}{ Hemoglobin level } \\
\hline Normal & $68(73.9)$ & $24(26.1)$ & \\
\hline Abnormal & $44(91.7)$ & $4(8.3)$ & \\
\hline \multicolumn{4}{|l|}{ Pulse rate } \\
\hline Normal & $86(82.7)$ & 18(17.3) & 0.0127 \\
\hline Abnormal & $26(72.2)$ & $10(27.8)$ & 0.176 \\
\hline \multicolumn{4}{|l|}{ Age group } \\
\hline Neonates & $43(64.2)$ & $24(35.8)$ & \\
\hline$>1$ month & $69(94.5)$ & $4(5.5)$ & 0.0001 \\
\hline
\end{tabular}

$p$ value $<0.05$ was significant

Normal sodium levels are between 135 to $145 \mathrm{mEq} / \mathrm{L}$

Normal potassium levels range between 3.5 and $5.5 \mathrm{mEq} / \mathrm{L}$ anomaly that is on the rise worldwide, is a significant burden in this region. Intussusception has maintained its burden as one of the commonest abdominal surgical emergencies not only in this region but also in most parts of the world $(5,7,8)$.

The complication rate was higher in this study than in other studies $(2,9)$. Patients who developed complications stayed significantly longer in hospital. This long hospital stay could be because patients with surgical complications underwent another operation to solve the complication, or due to prolonged antibiotic therapy for some complications like septicaemia. Similar results have been reported by other authors (10). Also, patients with gastroschisis stayed longer in hospital due to having staged procedures in theatre at different times. The mortality rate in this study was also higher than in other studies $(2,9)$. The highest mortality was in neonates, without any significant gender difference. Lack of adequate total parenteral nutrition and paediatric critical care facilities coupled with their low immunity and poor physiological body reserves in the face of serious congenital and acquired disorders could be responsible for the high mortality and morbidity in children $(2,11)$. Most patients presented late ( $>24$ hours). The effect of late presentation can be noted by the higher mortality rate compared with other studies. However, presentation of $<24$ hours had a statistically positive correlation with mortality. This might be due to the low number of patients presenting before 24 hours and the rush to operate on them without sufficient resuscitation, and also to poor monitoring of these patients with the assumption that their vital signs and electrolytes had been normal before surgery. Other factors affecting mortality were age $<2$ years, anemia, ASA classification grade 3 and 4 , and deranged potassium levels.

Despite correction of the deranged potassium levels (both high and low) before surgery, these had a positive correlation with mortality $(\mathrm{p}<0.0005)$. Stabilizing electrolytes before taking patients to theatre is a basic regulation worldwide. Emphasis should be on early presentation of patient to avoid severe electrolyte derangements and aggressive resuscitation $(2,5)$, and monitoring from admission till discharge. Neonates have intrinsic challenges like increased susceptibility to infections, hypocalcaemia, hypoglycemia and poor cardiovascular reserves related to the transition from fetal to post-natal life. Trauma of surgery distorts the delicate balance, making them susceptible to sepsis, dyselectrolytemias, anemia and nutritional deficits, and respiratory complications (2). 


\section{Conclusion}

The most common aetiologies of NTCASE at MTRH are intussusception and gastroschisis. Mortality and morbidity rates are clinically high. Factors correlated with mortality in patients with NTCASE are deranged potassium levels, ASA classification grade 3 and 4, neonates, normal hemoglobin level, duration of illness, presentation $<24$ hours, and development of complications. Increased morbidity and mortality in children with non-traumatic abdominal surgical emergencies at MTRH can be reduced by considering the factors associated with them.

\section{References}

1. Uba AF, Chirdan LB, Ituen AM, et al. Typhoid intestinal perforation in children: A continuing scourge in a developing country. Pediatr Surg Int. 2007; 23(1):33-9.

2. Ademuyiwa A, Bode C, Opeoluwa A, et al. Non-traumarelated paediatric abdominal surgical emergencies in Lagos, Nigeria: Epidemiology and indicators of survival. Niger Med J. 2012; 53(2):76-9.

3. Fisher RA. On the interpretation of $\chi^{2}$ from contingency tables, and the calculation of P. J Royal Stat. Soc. 1922; 85(1):87-94.
4. Pujari A, Methi RN, Khare N. Acute gastrointestinal emergencies requiring surgery in children at Indira Gandhi Government Medical College and Hospital, Nagpur. Afr J Paediatr Surg. 2008; 5(2):61-4.

5. Lukman O, Abdur-Rahman O, Adeniran J, et al. Paediatric surgical abdominal emergencies in a north central Nigerian centre. Ann Pediatr Surg. 2012; 8(2):25-8.

6. Abantanga $\mathrm{F}$, Lakhoo $\mathrm{K}$. Inguinal and femoral hernias and hydroceles. In: Ameh E, Bickler S, Lakhoo K, et al., editors. Paediatric surgery: A comprehensive text for Africa. Seattle, Washington: Global-Help; 2010; pp. 358-65.

7. Shiekh KA, Baba AA, Ahmad SM, et al. Mechanical small bowel obstruction in children at a tertiary care centre in Kashmir. Afr J Paediatr Surg. 2010; 7(2):81-5.

8. Kuremu RT. Childhood intussusception at the Moi Teaching and Referral Hospital, Eldoret: Management challenges in a rural setting. East Afr Med J. 2004; 81(9):443-6.

9. Rajendra KG, Budhwani KS, Dhirendra KS. Exploratory laparotomy for acute intestinal conditions in children: A review of 10 years of experience with 334 cases. Afr J Paediatr Surg. 2011; 8(1):62-9.

10. Chalya PL, Mabula JB, Chandika AB, et al. Dynamic bowel obstruction: Etiology, clinical presentation, management and outcome at Bugando Medical Centre, Mwanza, Tanzania. Tanzan J Health Res. 2014; 16(1):38-46.

11. Jumbi G, Tenge R, Khwa-Otsyula B, et al. Epidemiology of acute intestinal obstruction in Uasin Gishu County, Kenya. ECAMJ. 2017; 3(1):21-5. 